\title{
Optimization of Extraction Parameters of Phenolic Compounds from Sarcopoterium spinosum Leaves by Response Surface Methodology
}

\author{
Ceren Sunguc $^{1}$, Oguz Bayraktar ${ }^{2 *}$, Ipek Erdogan ${ }^{1}$, Mehmet Emin Uslu ${ }^{3}$ \\ ${ }^{I}$ Department of Biotechnology and Bioengineering, Faculty of Engineering, Izmir Institute of Technology, 35430 Izmir, Turkey \\ ${ }^{2}$ Department of Chemical Engineering, Faculty of Engineering, Ege University, 35040 Izmir, Turkey \\ ${ }^{3}$ Department of Biomedical Equipment Technology, Soma Vocational School, Celal Bayar University, 45500 Manisa, Turkey

\section{A R T I C L E I N F O}

A B S T R A C T

\section{Research Article}

Received 25 February 2017

Accepted 28 June 2017

\section{Keywords}

Extraction parameters

Hyperoside

Isoquercetin

Phenolic content

Prickly shrubby burnet

*Corresponding Author:

E-mail: oguz.bayraktar@ege.edu.tr

\begin{abstract}
The shrublands are very common in Urla-Çeşme-Karaburun peninsula located in the western point of Turkey. Prickly shrubby burnet (Sarcopoterium spinosum L.) is one of the common weed which has intensive thorns making its consumption for the local domestic animals. However, Sarcopoterium spinosum is a valuable and common medicinal plant in the Mediterranean region. Crude extract of $S$. spinosum leaves exhibited higher antioxidant activity, as $3143.5 \pm 238.5 \mu \mathrm{M}$ TEAC (Trolox Equivalent Antioxidant Capacity)/g dry weight (DW), when compared to other medicinal plants found in the literature. The aim of this study was to determine the effect of extraction parameters on the content and biological activity of the extract by response surface methodology (RSM) as well as to identify its major compounds. High Performance Liquid Chromatography (HPLC) was employed to investigate the phenolic content of $S$. spinosum extract. The composition of the phenolic contents including hyperoside and isoquercetin, the latter being the major component, in S. spinosum extract has been shown for the first time by HPLC. Antimicrobial activity of $S$. spinosum extract, identified by minimum inhibition concentration (MIC) assay, indicated that the crude extract had antifungal activity against Candida albicans.
\end{abstract}

DOI: https://doi.org/10.24925/turjaf.v5i7.792-799.1195

\section{Introduction}

Plant derived natural phenolic compounds have potentials to be utilized in many different industries including medical, cosmetic, food and pharmaceutical. Phenolic compounds are known for their antioxidant activity which refers to ability to scavenge free radicals that cause diseases related with oxidative stress. In addition, bioactive compounds isolated from medicinal plants can effectively be used for the development of new drugs in pharmaceutical industry (Cragg and Newman, 2005; Bayraktar et al., 2016). S. spinosum, an endemic species, having high antioxidant capacity and phenolic content can easily be found in the Mediterranean Region (Georgios et al., 2007; Bar and Negoitza, 2007; Domenico et al. 2007; Ronel et al., 2007; Al-Mustafa and Al-Thunibat, 2008). S. spinosum has been used in complementary medicine for preventing the symptoms of several diseases such as diabetes, pain, digestive problems or cancer (Yaniv, 2007; Rao et al., 2010). It was especially considered as a potential anti-diabetic drug (Reher et al., 1991a; Reher et al., 1991b; Reher and Budesinsky, 1992; Rosenzweig et al., 2007), exhibiting an effect similar to insulin for the uptake of glucose in hepatocytes (Kasabri et al., 2011), increasing insulin secretion in vitro, and having an improved glucose tolerance in vivo (Smirin et al., 2010).

In the literature, phytochemical composition of the extracts mostly from the roots of Sarcopoterium spinosum was investigated. The antidiabetic activities of the extracts from the roots of Sarcopoterium spinosum were usually attributed to the presence of triterpenes and tannins in the root extracts (Reher and Budesinsky, 1992; Lobna et al., 2013). Recently, phenolic and flavonoid contents in extracts from the aerial parts of Sarcopoterium spinosum were evaluated for their antimicrobial and cytotoxic activities (ElHawary et al., 2016).

In the present study the objective was to determine the conditions for the optimum extraction of phenolic compounds with antioxidant and antimicrobial activity by using response surface methodology (RSM). It was also aimed to identify the major compounds in ethanolic extract of S. spinosum leaves and establish a relation with the antioxidant and antimicrobial activity. Although it has been proposed that $S$. spinosum would be used in traditional medicine to the best of our knowledge, the presence of isoquercetin and hyperoside within ethanolic extract of $S$. spinosum leaves has not been reported earlier. 


\section{Materials and Methods}

\section{Preparation of Crude Extract}

Aerial parts of S. spinosum grown in Urla, İzmir were collected in spring during the flowering period. The leaves of $S$. spinosum were dried at room temperature. After grinding the dried leaves the powder form of plant material was obtained. Extraction was performed in aqueous ethanol solution by changing several parameters of interest including extraction time, solvent concentration and liquid-solid ratio at constant initial amount of plant material (10 g) and agitation speed (180 rpm). A set of experiments determined by design of experiment software (Minitab Inc., State College, PA, USA) were carried out. Extract was first filtered and then evaporated under vacuum at $35^{\circ} \mathrm{C}$ to remove ethanol. Extract was obtained after centrifuging at $5000 \mathrm{rpm}$ for 10 min to remove solid particles, followed by lyophilization.

Determination of Total Phenolic Content and Total Antioxidant Capacity

Folin-Ciocalteu method (Singleton and Rossi, 1965; Ainsworth and Gillespie, 2007) was used to determine the total phenol contents of extracts obtained from $S$. spinosum leaves. Total phenol content was given in terms of Gallic acid equivalents (GAE). Trolox, a standard antioxidant, was used for the standard calibration curve to compare the capability of the extract to scavenge ABTS radical cation (Re et al., 1999).

\section{High Performance Liquid Chromatography (HPLC)} Analysis.

The HPLC analysis was performed to identify the composition of the extract by comparison with standards of gallic acid, catechin, rutin, hyperoside and isoquercetin (Ying et al., 2009). The HPLC equipment used was Agilent 1100 equipped with the diode array detector (DAD). For chromatographic analysis phosphoric acid/water (1: 99, v/v) and (B) tetrahydrofuran: acetonitrile $(5: 95, \mathrm{v} / \mathrm{v})$ were used as mobile phases with a flow rate of $1 \mathrm{ml} / \mathrm{min}$. LiChrospher ${ }^{\circledR} 100 \mathrm{RP}-\mathrm{C}_{18}$ column $(4 \times 250 \mathrm{~mm})$ at $30^{\circ} \mathrm{C}$ was used as stationary phase. The changes in absorbance values at 280 and $360 \mathrm{~nm}$ were monitored during analyses.

\section{Thin Layer Chromatography (TLC)}

Mobile phase consisted of ethyl acetate: formic acid: acetic acid: water (100: 11: 11: 26). TLC plate was loaded with samples of standards and crude extract dissolved in appropriate solvents. After developing, TLC plates were dried in the oven at $100^{\circ} \mathrm{C}$ for 3 minutes. The plates were first sprayed with $1 \%$ natural product reagent (NP), then with 5\% ethanolic polyethylene glycol (PEG) solution. UV light $(366 \mathrm{~nm})$ under dark room was used for the observation of bands on the TLC plates.

\section{Minimum Inhibition Concentration (MIC) Assay}

For antimicrobial studies, Lyophilized forms of Candida albicans(ATCC-64548), Escherichia coli(NRRL B-3008)and Staphylococcus epidermidis(ATCC-12228) were obtained from American Type Culture Collections (ATCC). MIC was employed to determine the critical concentration of the extract for antimicrobial effect. Potato broth was used as the inoculation medium for Candida albicans. Nutrient broth was used for Escherichia coli and Staphylococcus epidermidis. The cultures were incubated overnight at $37^{\circ} \mathrm{C}$. Microbial count was determined by 0.5 McFarland turbidity standards after overnight incubation. Crude extract was subjected to serial dilution with appropriate media. The turbidity measurements at $600 \mathrm{~nm}$ for growth kinetics of individual strains were carried out in triplicate using a micro plate reader (Varioskan) at $37^{\circ} \mathrm{C}$ for $24 \mathrm{~h}$, on an agitated platform at $60 \mathrm{rpm}$. The concentrations that inhibit the growth of each strain were reported as MIC values $(\mathrm{mg} / \mathrm{ml})$.

\section{Disc Diffusion Assay}

The antimicrobial activities of $S$. spinosum extract having the highest and lowest antioxidant capacity and total phenolic content were obtained with disc diffusion assays. Fresh culture for each microorganism was prepared as explained in MIC assay part described above. Followed by determination of microbial count after overnight incubation, appropriate amount of microorganisms were spread on agar plates. Amount of inoculum for $S$. epidermidis and E. coli were $1 * 10^{6} \mathrm{CFU}$ respectively, while it was $1 * 10^{4} \mathrm{CFU}$ for $C$. albicans. After colonization, sterile discs without any sample (blank) were placed on plates inoculated with the microorganism of interest. The sterile blank discs were soaked with the $15 \mu \mathrm{l}$ of aqueous extracts $(50 \mathrm{mg} / \mathrm{ml})$ obtained at different extraction parameters. The diameters of the inhibition zones around the discs were recorded in millimeter $(\mathrm{mm})$ after all plates were incubated at $37^{\circ} \mathrm{C}$ for 24 hours. Except gram positive $S$. epidermidis the plates were sprayed with p-Iodonitrotetrazolium violet (INT) solution to form pink stain as a result of reaction with the metabolites produced by the microorganisms. The formation of pink colour enhanced the visibility the viable colonies

\section{Results and Discussion}

\section{Optimization Of Extract Preparation}

The determination of the optimum parameters for the natural compound extraction from plant material has been the focus of many comprehensive studies (Montgomery, 2008; Choi et al., 2014; Lingzhu et al., 2015). Size of the material, solvent type, extraction time, liquid-solid ratio can be listed as several important parameters for the extraction of phenolic compounds from plant materials. In this study, the effects of several selected parameters on yield, total phenol content (TPC) and Trolox equivalent antioxidant capacity (TEAC) of extracts were investigated using response surface methodology (Bezerra et al., 2008).

For the central composite design of experiments the coded and deleted levels of independent parameters along with their lower, middle and upper design values are 
tabulated in Table 1. Ethanol concentration $\left(\mathrm{x}_{1}\right)$, extraction time $\left(\mathrm{x}_{2}\right)$ and liquid-solid ratio $\left(\mathrm{x}_{3}\right)$ were selected as parameters for the extraction of phenolic compounds from $S$. spinosum leaves.

The second order surface response method reperesented with equation 1 below was used to fit the experimental data (Montgomery, 2008).

$Y=\beta_{0}+{ }_{i} \cdot x_{i}+{ }_{i i} \cdot x_{i}^{2}+\sum_{i j} \cdot x_{i} \cdot x_{j}$

The measured response variables are represented with $Y$. The experimental model in equation 1 consists of a constant $\beta_{0}$, linear, quadratic and interactive coefficients $\beta_{\mathrm{i},} \beta_{\mathrm{ii}}$ and $\beta_{\mathrm{ij}}$, respectively. $x_{i}, x_{j}$ and $x_{i j}$ are the levels of the independent variables. The response surface graphs were plotted by varying two variables and keepingthe others constant at the central point. The decission for the statistical significance test was made with a confidence interval level of $95.0 \%$.

Effect of different conditions in extraction process of phenolic compounds from $S$. spinosum was compared in terms of TPC and TEAC assays. The experimental data and results predicted using model equation obtained central composite experimental design analysis are tabulated in Table 2. After fitting data into model equation regression coefficients $\left(r^{2}\right)$ for total antioxidant capacity $\left(Y_{1}\right)$ and total phenol content $\left(Y_{2}\right)$ were determined as 0.88 and 0.87 , respectively.

The correlation between total antioxidant capacity of extract and extraction parameters is given in equation 2 . Similarly equation 3 describes the realationship between total phenolic content of extract and extraction parameters.

$$
\begin{aligned}
& Y_{l}=2805.7+24.3 x_{1}-193.6 x_{2}-80.7 x_{3}+7.55 x_{2}{ }^{2}-0.35 x_{1} x_{3}+3.85 x_{2} x_{3} \\
& Y_{2}=172.7+1.139 x_{1}+5.417 x_{3}
\end{aligned}
$$

When the extraction time and liquid-solid ratio were used to obtain the response surface plots, it was revealed that those parameters exhibited negative quadratic effect on the total antioxidant capacity of the extract as seen in Figure 1-A. If the interaction of ethanol concentration with the liquid-solid ratio was analysed, increasing ethanol concentration positively affected and caused an increase in the total antioxidant capacity as shown in Figure 1-B.

Table 1The levels and values of independent extraction parameters used for the response surface analysis

\begin{tabular}{l|lccc}
\hline \multirow{2}{*}{ Independent variable } & & \multicolumn{3}{c}{ Coded levels } \\
\cline { 3 - 5 } & & Units & -1 & 0 \\
\cline { 3 - 4 } & & 10 & Uncoded levels \\
\hline Ethanol concentration, $\mathrm{x}_{1}$ & $\%(\mathrm{v} / \mathrm{v})$ & 2 & 50 & 90 \\
Extraction time, $\mathrm{x}_{2}$ & Hour & 10 & 20 & 14 \\
Liquid-solid ratio, $\mathrm{x}_{3}$ & $\mathrm{ml} / \mathrm{mg}$ & 8 & 30 \\
\hline
\end{tabular}

Table 2 The experimental data and results predicted using model equation for total phenolic content and total

\begin{tabular}{|c|c|c|c|c|c|c|c|}
\hline \multirow{2}{*}{ Test set } & \multirow{2}{*}{$\mathrm{x}_{1}$} & \multirow{2}{*}{$\mathrm{x}_{2}$} & \multirow{2}{*}{$\mathrm{x}_{3}$} & \multicolumn{2}{|c|}{ TEAC ( $\mu$ mole /g DW $)$} & \multicolumn{2}{|c|}{ TPC (mg GAE /g DW) } \\
\hline & & & & Experimental* & $\mathrm{Y}_{1}$, Predicted $^{* *}$ & Experimental $^{*}$ & $\mathrm{Y}_{2}$, Predicted $* * *$ \\
\hline 1 & 10 & 2 & 10 & $1995.7 \pm 353$ & 1927.1 & $255.5 \pm 14$ & 238.3 \\
\hline 2 & 90 & 2 & 10 & $3033.0 \pm 164$ & 3592.7 & $334.2 \pm 9$ & 329.4 \\
\hline 3 & 10 & 14 & 10 & $1790.0 \pm 232$ & 1515.3 & $205.4 \pm 12$ & 238.3 \\
\hline 4 & 90 & 14 & 10 & $2473.9 \pm 312$ & 3180.9 & $332.5 \pm 6$ & 329.4 \\
\hline 5 & 10 & 2 & 30 & $2125.4 \pm 223$ & 397.7 & $301.4 \pm 10$ & 346.6 \\
\hline 6 & 90 & 2 & 30 & $2174.5 \pm 243$ & 1503.3 & $386.3 \pm 8$ & 437.7 \\
\hline 7 & 10 & 14 & 30 & $2420.6 \pm 186$ & 909.9 & $266.6 \pm 16$ & 346.6 \\
\hline 8 & 90 & 14 & 30 & $2960.4 \pm 274$ & 2015.5 & $312.6 \pm 20$ & 437.7 \\
\hline 9 & 10 & 8 & 20 & $3143.5 \pm 238$ & 915.7 & $280.1 \pm 15$ & 292.4 \\
\hline 10 & 90 & 8 & 20 & $2096.5 \pm 125$ & 2301.3 & $385.1 \pm 18$ & 383.6 \\
\hline 11 & 50 & 2 & 20 & $2239.5 \pm 155$ & 1855.2 & $337.5 \pm 15$ & 338.0 \\
\hline 12 & 50 & 14 & 20 & $2379.1 \pm 238$ & 1905.4 & $276.6 \pm 7$ & 338.0 \\
\hline 13 & 50 & 8 & 10 & $2182.4 \pm 236$ & 2282.2 & $286.8 \pm 9$ & 283.8 \\
\hline 14 & 50 & 8 & 30 & $2253.9 \pm 123$ & 934.8 & $360.0 \pm 17$ & 392.2 \\
\hline 15 & 50 & 8 & 20 & $2148.3 \pm 232$ & 1608.5 & $259.6 \pm 22$ & 338.0 \\
\hline 16 & 50 & 8 & 20 & $1972.0 \pm 243$ & 1608.5 & $286.1 \pm 12$ & 338.0 \\
\hline 17 & 50 & 8 & 20 & $1981.7 \pm 177$ & 1608.5 & $305.0 \pm 18$ & 338.0 \\
\hline 18 & 50 & 8 & 20 & $2599.3 \pm 243$ & 1608.5 & $297.2 \pm 17$ & 338.0 \\
\hline 19 & 50 & 8 & 20 & $2127.5 \pm 238$ & 1608.5 & $262.1 \pm 8$ & 338.0 \\
\hline 20 & 50 & 8 & 20 & $2361.4 \pm 213$ & 1608.5 & $346.4 \pm 14$ & 338.0 \\
\hline
\end{tabular}
antioxidant capacity 
Table 3 The experimental data and results predicted using model equation for mass yield percentage

\begin{tabular}{|c|c|c|c|c|c|}
\hline \multirow{2}{*}{ Test set } & \multirow{2}{*}{$\mathrm{x}_{1}$} & & \multirow{2}{*}{$\mathrm{X}_{3}$} & \multicolumn{2}{|c|}{ Mass yield (\%) } \\
\hline & & & & Experimental* & $\mathrm{Y}_{3}$, Predicted $* *$ \\
\hline 1 & 10 & 2 & 10 & $8.6 \pm 0.5$ & 10.3 \\
\hline 2 & 90 & 2 & 10 & $8.2 \pm 0.7$ & 14.9 \\
\hline 3 & 10 & 14 & 10 & $17.5 \pm 0.4$ & 19.0 \\
\hline 4 & 90 & 14 & 10 & $17.5 \pm 1.2$ & 10.3 \\
\hline 5 & 10 & 2 & 30 & $7.2 \pm 0.5$ & 10.3 \\
\hline 6 & 90 & 2 & 30 & $11.9 \pm 0.6$ & 19.0 \\
\hline 7 & 10 & 14 & 30 & $19.7 \pm 0.7$ & 19.0 \\
\hline 8 & 90 & 14 & 30 & $18.4 \pm 0.2$ & 19.0 \\
\hline 9 & 10 & 8 & 20 & $21.0 \pm 0.4$ & 19.0 \\
\hline 10 & 90 & 8 & 20 & $20.4 \pm 1.0$ & 14.9 \\
\hline 11 & 50 & 2 & 20 & $15.0 \pm 0.6$ & 14.9 \\
\hline 12 & 50 & 14 & 20 & $14.6 \pm 0.5$ & 19.0 \\
\hline 13 & 50 & 8 & 10 & $18.1 \pm 0.8$ & 10.3 \\
\hline 14 & 50 & 8 & 30 & $11.0 \pm 0.1$ & 19.0 \\
\hline 15 & 50 & 8 & 20 & $19.3 \pm 0.2$ & 19.0 \\
\hline 16 & 50 & 8 & 20 & $11.2 \pm 0.8$ & 14.9 \\
\hline 17 & 50 & 8 & 20 & $8.8 \pm 0.4$ & 19.0 \\
\hline 18 & 50 & 8 & 20 & $12.0 \pm 0.5$ & 19.0 \\
\hline 19 & 50 & 8 & 20 & $19.0 \pm 0.3$ & 14.9 \\
\hline 20 & 50 & 8 & 20 & $13.8 \pm 1.1$ & 11.8 \\
\hline
\end{tabular}
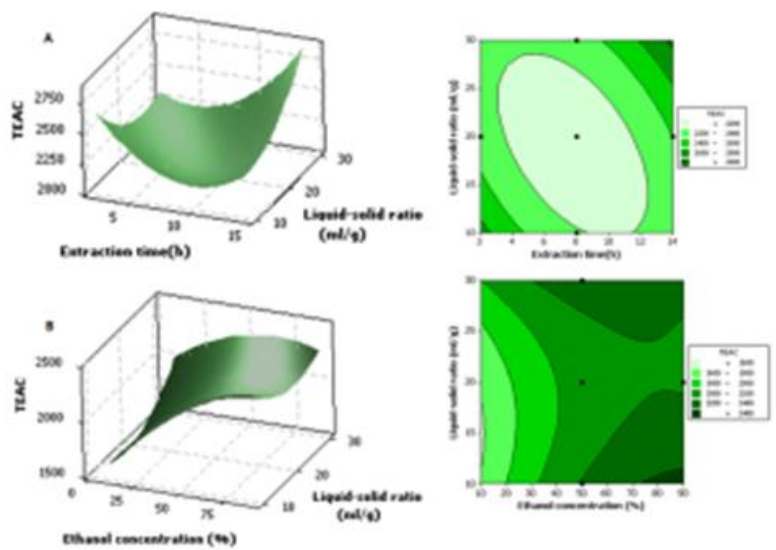

Figure 1 Response surface and contour graphs

A) for the effects of extraction time and liquid-solid ratio on total antioxidant capacity (Y1) at constant ethanol concentration of 50\%; B) for the effects of ethanol concentration and liquid-solid ratio on total antioxidant capacity (Y1) at constant extraction time of $8 \mathrm{~h}$.

Effects of extraction time and liquid-solid ratio on total phenol content $\left(\mathrm{Y}_{2}\right)$ were also subjected to surface response methodology. Response surface plots, obtained at fixed ethanol concentration of $50 \%$, exhibited that increase in extraction time affected total phenol content in negative manner, as depicted in Figure 2-A. Total phenol content was also significiantly affected with ethanol concentration, regardless of extraction time and liquidsolid ratio. Figure $2-\mathrm{B}$ and $2-\mathrm{C}$ indicated the significant increase in the amount of phenolic compounds with increasing ethanol content, which can be attributed to the enhanced solubility of phenolic compounds in aqueous ethanol, as expected. This result was in accordance with the experimental results indicating significant increase in total antioxidant capacity with increasing ethanol concentration.
Extraction parameters were also tested to establish a relationship for mass yield. Table 3 denotes that experimental data obtained at different extraction parameters including ethanol concentration, extraction time and liquid-solid ratio, were in accordance with predicted values with a good regression coefficient $\left(r^{2}=0.91\right)$. Equation 4 shows the relationship between mass yield and extraction parameters.

$$
Y_{3}=11.827+0.343 x_{1}-0.0037 x_{1}^{2}
$$

Some effective parameters for mass yield of the extraction process are temperature, time and solvent polarity. However, the main motivation behind this study was to determine the optimum conditions for extraction process resulting in extracts with highest antioxidant capacity due to its high phenolic content. As seen in the results of response surface and contour plots (Figure 3-A), mass yield percentage of extract was significantly affected with varying ethanol concentration. At 40 to $50 \%$ ethanol concentration and relatively high liquid-solid ratio (approx. 30) it was possible to reach more than $20 \%$ mass yield.

The results shown in Figure 3-B indicated that both extraction time and ethanol concentration had significant effect on mass yield percentage of extraction process. Mass yield percentage of extract was significantly affected with varying ethanol concentration. At 40 to $50 \%$ ethanol concentration and 8 to 10 hour extraction time it was possible to reach more than $18 \%$ mass yield.

\section{Compositional Analysis of the Crude Extract}

Identification of the phenolic compounds in the extract was performed by HPLC analyses. The retention times for individual phenolic compounds along with their 
corresponding UV spectral data are given in Figure 4 and 5. The retention times of major phenolic substances and their UV absorbance spectra were confirmed using corresponding reference standards. In the HPLC chromatogram of the extract recorded at $270 \mathrm{~nm}$, two peaks detected at the retention times 16.99 (peak 4) and 18.04 (peak 5), were identified as hyperoside and isoquercetin, respectively.
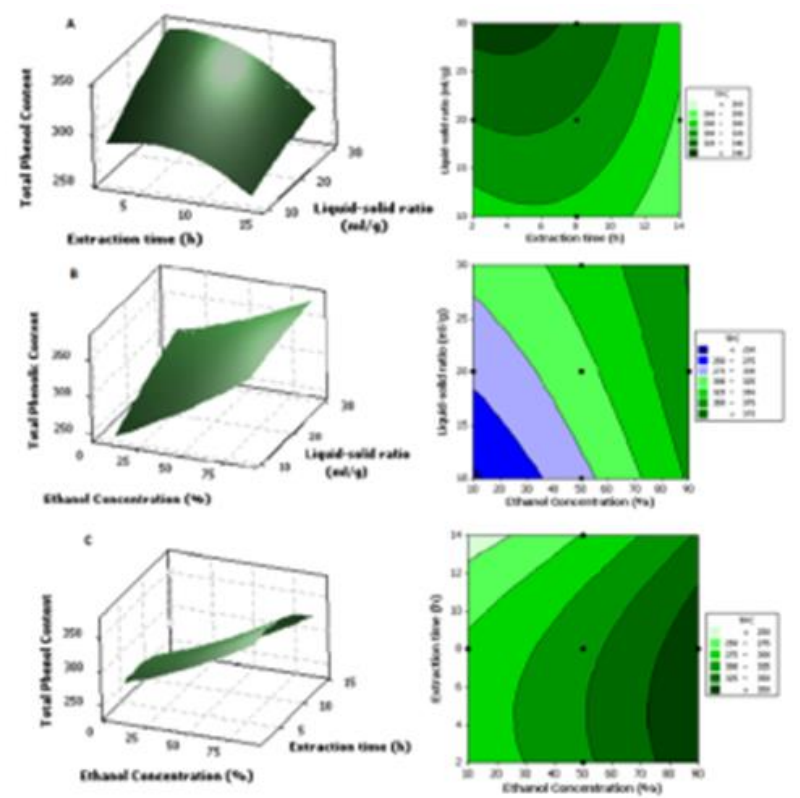

Figure 2 Response surface and contour graphs A) for the effects of extraction time and liquid-solid ratio on total phenol content (Y2) at constant ethanol concentration of 50\%; B) for the effects of ethanol concentration and liquid-solid ratio on total phenol content (Y2) at constant extraction time of $8 \mathrm{~h} ; \mathrm{C}$ ) for the effects of extraction time and ethanol concentration on total phenol content (Y2) at at constant liquid-solid ratio of 20
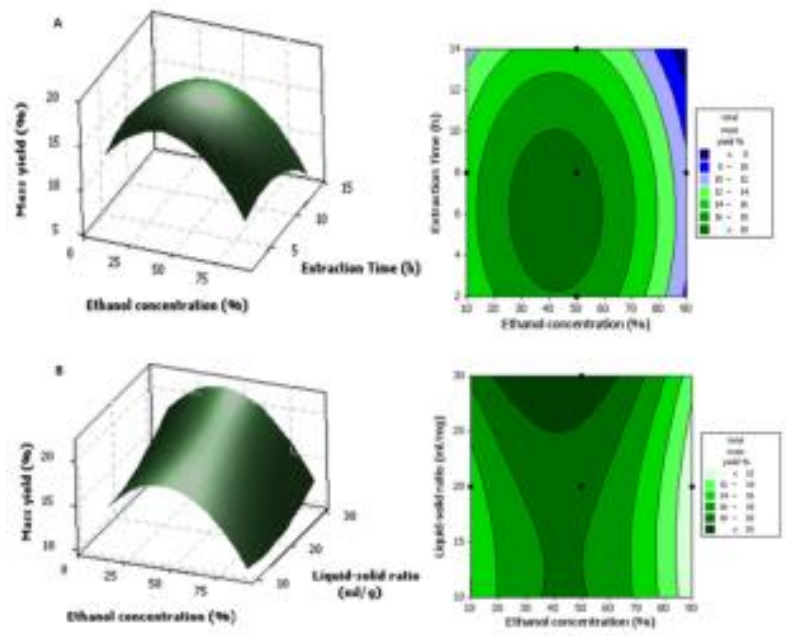

Figure 3 Response surface and contour graphs

A) for the effects of ethanol concentration and liquid-solid ratio on mass yield percentage (Y3) at constant extraction time of $8 \mathrm{~h}$; B) for the effects of extraction time and ethanol concentration on mass yield percentage (Y3) at constant liquid-solid ratio of 20.
UV spectra and retention time of compounds were confirmed with commercially available standards. Compound 1 shared similar UV spectra and retention time (Rt) with gallic acid, while compound 2 exhibited characteristic $\mathrm{UV}_{\max }$ at $220 \mathrm{~nm}$ and $280 \mathrm{~nm}$ for catechin, as seen in Figure 5. The amount of catechin in crude extract was calculated as $17.8 \mathrm{mg}$ per $\mathrm{g}$ dried weight of extract. The retention time and UV spectra of compound 3 in the chromatogram had been confirmed by reference standard of rutin.

Earlier, presence of catechin was shown using droplet countercurrent chromatography (DCCC) (Reher et al., 1991b). Later, catechin and epicatechin were also identified by liquid chromatography and mass spectroscopy (LC/MS) in aqueous extract of $S$. spinosum (Smirin et al., 2010). Recently, using HPLC analyses presence of catechin, epicatechin, gallic acid and rutin in methanolic extract of $S$. spinosum leaves were reported (El-Hawary et al., 2016). The UV spectra and retention time of compound 4 and 5 were in good match with those of hyperoside (Rt, $16.9 \mathrm{~min}$ ) and isoquercetin (Rt, 17.8 min). Both compounds showed their $\mathrm{UV}_{\max }$ at $360 \mathrm{~nm}$, specifically. In addition to UV spectral characterization, the identification of hyperoside and isoquercetin was further confirmed by spiking their commercial standards into extract solution before analysis. The amount of hyperoside was calculated as $12.9 \mathrm{mg}$ per $\mathrm{g}$ dried weight of extract. Isoquercetin, the major compound of $S$. spinosum extract, was $36.6 \mathrm{mg}$ per g dried weight of extract.

Thin layer chromatography was employed for confirmation of HPLC analysis. After staining, phenolic compounds in the extract caused a formation of distinctively separated bands on the TLC plate (Figure 6). Standards of rutin, hyperoside, isoquercetin and gallic acid were in accordance with the compounds in the extract of $S$. spinosum, indicating their presence.

The novelty of our research was the identification of the isoquercetin and hyperoside content of S. spinosum extract. The presence of isoquercetin and hyperoside were confirmed with the results of HPLC. There is no supportive published data which can identify the content of S. spinosum in the extract with these compounds. For this reason, all compound identification was carefully studied and confirmed by many different trial and repetition. In the literature, there is no report on the presence of these natural compounds in S. spinosum. In this study, especially two main compounds (hyperoside and isoquercetin) were successfully identified in the ethanolic extract of $S$. spinosum leaves.

\section{Antimicrobial Activity}

Disc diffusion assays were performed for $S$. spinosum extract to observe the differences resulting from difference in extraction parameters as well as to compare these results with the standard extraction conditions that were $70 \%$ aqueous ethanol as extraction solvent, 20:1 liquid-solid ratio and 4 hour extraction time. Inhibition zones for the extracts were observed by INT staining. 


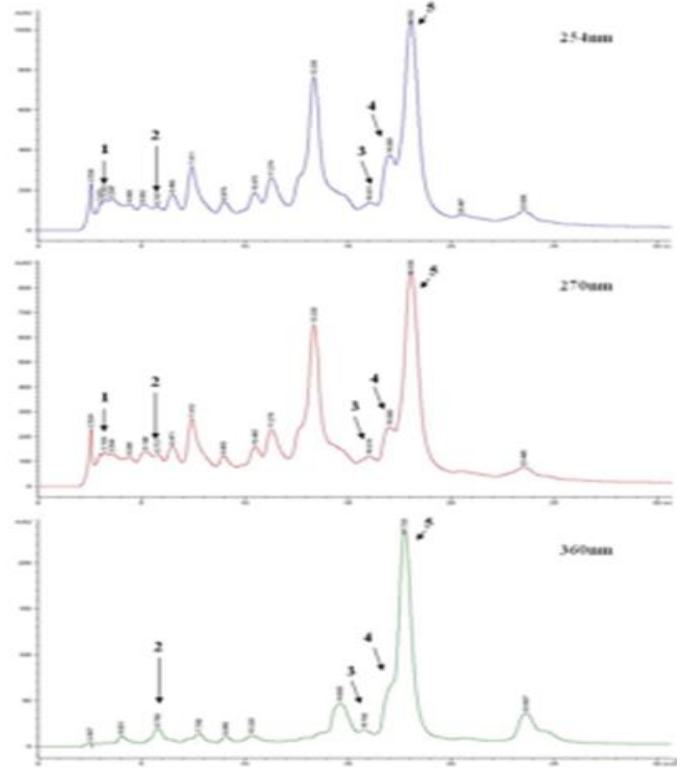

Figure 4 HPLC chromatograms recorded at 254, 270 and $360 \mathrm{~nm}$ for the S. spinosum extract.

Compounds are denoted with numbers: 1 - gallic acid, 2- catechin, 3rutin, 4-hyperoside and 5-isoquercetin.

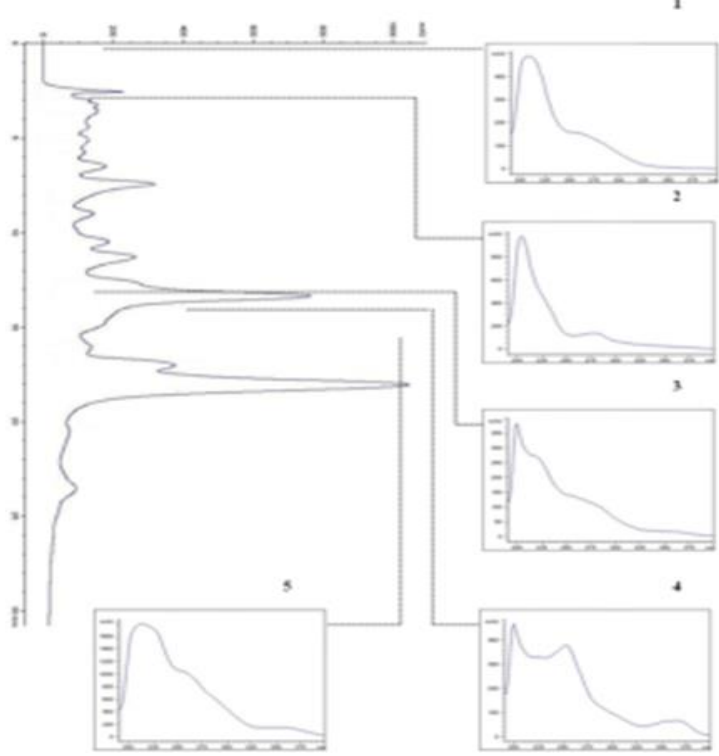

Figure 5. HPLC profile with the UV spectra of S. spinosum extract for peak

1(gallic acid), peak 2 (catechin), peak 3(rutin), peak 4 (hyperoside) and peak 5 (isoquercetin).

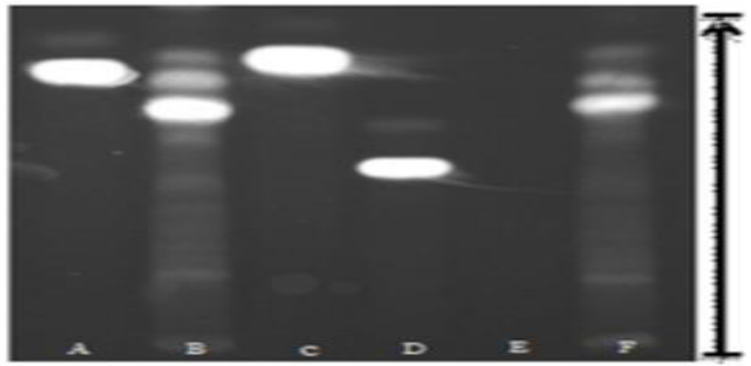

Figure 6 The picture of the TLC plate.

Samples are S. spinosum extract (denoted by B and F) and standards (hyperoside; A, isoquercetin; C, rutin; D and gallic acid; E). Arrow denotes the direction of the run.
Antimicrobial effects of the standard extract against $S$. epidermidis were not clearly identified because staining procedure was not compatible with the gram positive bacteria. S. spinosum extract formed an inhibition zone at moderate level against $S$. epidermidis. In addition, $C$. albicans and $E$. coli were more sensitive to the plant extract than $S$. epidermidis, indicated by determined inhibition zones depicted in Figure 7.

$S$. spinosum extracts obtained at different extraction parameters also exhibited antimicrobial activity against all species, among which $C$. albicans was the most susceptible (Figure 8). Extracts were chosen according to antioxidant activity and total phenol content they possessed. The extract with the highest antioxidant activity coded as test $\# 9$, the highest phenolic content coded as test \#6 and the lowest value for both coded as test \#3 were taken into antimicrobial tests.

Quantitative analyses of the antimicrobial activity of S.spinosum extract against E.coli, S. epidermidis and $C$. albicans were evaluated by MIC assays (Figure 9). Crude extract obtained at standard extraction conditions of $70 \%$ aqueous ethanol at 20:1 liquid-solid ratio during 4 hours at room temperature exhibited its inhibitory effect on $C$. albicans at $2.5 \mathrm{mg} / \mathrm{ml}$, which can be considered as the MIC value for this specie. Low concentrations of the extract exerted bacteriostatic effect on $E$. coli and $S$. epidermidis. It was also stated that ethanolic extract of $S$. spinosum exhibited better antifungal effect against $C$. albicans, when compared with its antibacterial effect against $E$. coli by determination of inhibition zone diameters (Ali-Shtayeh et al.,1998). They also revealed that ethanolic extracts overall exerted better antimicrobial effects in comparison with aqueous extract. Ethanolic extract of $S$. spinosum can be considered as a potential antifungal overall. In another study performed by AliShtayeh et al. (2013), 56 plant species were screened for antimicrobial activity against 16 strains including $P$. acnes, E. coli, K. pneumonia, P. vulgaris, P. aeruginosa and S. aureus. Sarcopoterium spinosum was regarded as one of the most active plants against all used species according to MIC, inhibition zone diameter and minimum bactericidal concentration (MBC) results due to its polyphenolic nature and flavonoid content. S. spinosum also exhibited significant inhibitory activity against $C$. albicans in terms of inhibitory zone diameter when compared with Arbutus pavarii, which also has a polyphenolic nature as determined by El-Hawary et al. (2016), and commercial amphotericin B. As antimicrobial effect was found related with polyphenolic nature of the crude extract (Reyes-Munguia et al., 2016), antimicrobial activity of $S$. spinosum is in accordance with its high polyphenolic content. Dibas et al. (2017) performed screening for antioxidant activity, total phenolic and flavonoid contents of five medicinal selected plants of Jordan. Among them S. spinosum was found to have highest antioxidant activity and highest ferric reducing power which is an indicator of its phenolic content with respect to ascorbic acid. These findings exhibited correlation with the high phenolic and flavonoid content of S. spinosum. 


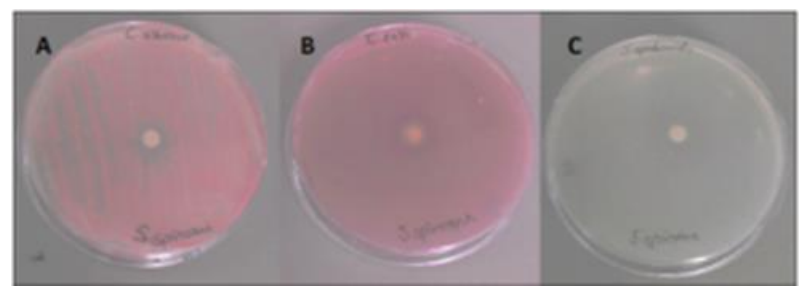

Figure 7 Disc diffusion assays for S. spinosum extract Inhibition zones for C. albicans (A), E. coli (B), S. epidermidis (C).

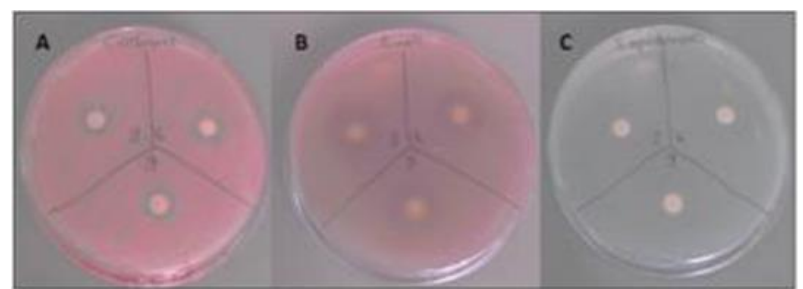

Figure 8 Disc diffusion assays for S. spinosum extract Inhibition zones of plant extracts (\#3, \#6, \#9) for C. albicans (A), E. coli (B), S. epidermidis (C). Extracts were obtained at different extraction parameters.
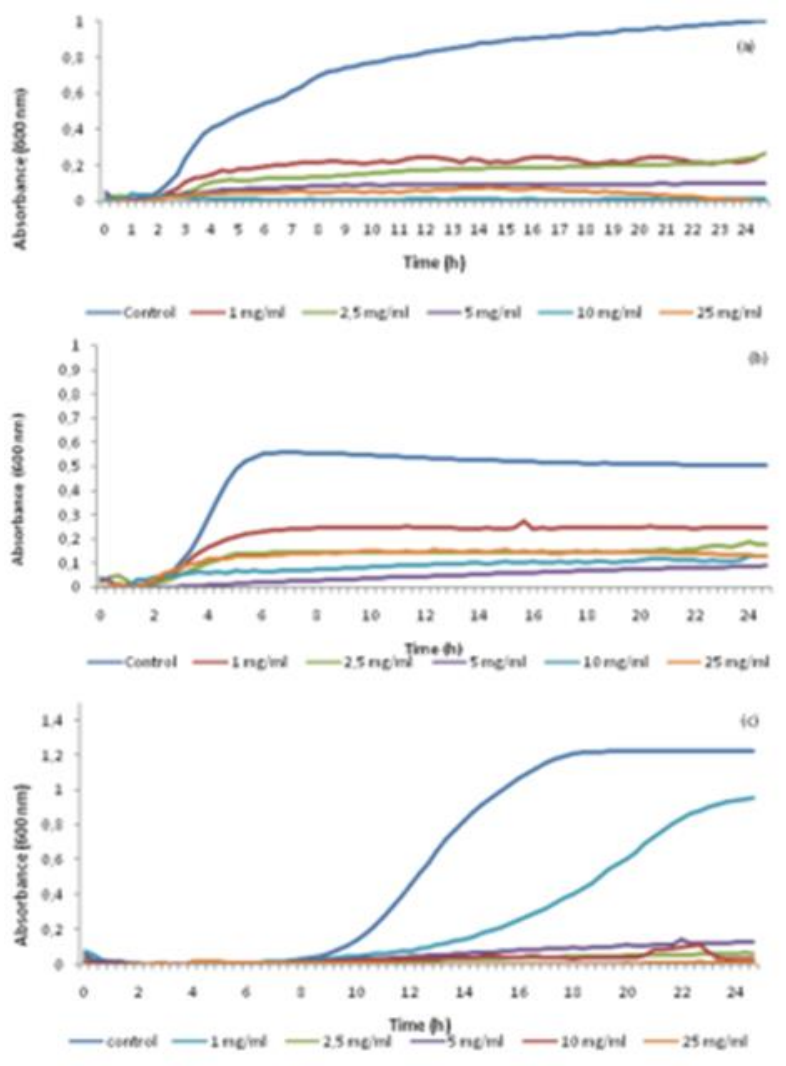

Figure 9 The growth curve of (a) E. coli, (b) S. epidermidis and (c) C. albicans in liquid media including different concentrations of S. spinosum extract.

\section{Conclusions}

Sarcopoterium spinosum used in this study is a commonly used folk medicine in the Mediterranean region. Despite its use in diabetic regulation, there is limited information about $S$. spinosum compounds. Effects of selected extraction parameters on mass yield, total phenol content, total antioxidant and antimicrobial activities and phenolic composition of ethanolic extract of S. spinosum were investigated. Ethanol content was found as the most prominent parameter to obtain high phenolic content as well as high antioxidant capacity while extraction time affected these properties in negative manner. $S$. spinosum extract had relatively high antioxidant activity when compared with extracts of various plants studied in the literature. The presence of hyperoside and isoquercetin in S.spinosum leave extract were detected using by HPLC. To the best of our knowledge, this is the first report demonstrating the presence of isoquercetin and hyperoside within ethanolic extract of $S$. spinosum leaves. High phenolic content and antioxidant capacity also affected antimicrobial activities indicated as dominant antifungal and bacteriostatic properties. It may be possible to use the ethanolic extract of S.spinosum as an effective therapeutic agent.

\section{References}

Ainsworth EA, Gillespie KM. 2007. Estimation of total phenolic content and other oxidation substrates in plant tissues using Folin-Ciocalteu reagent. Nat Protoc., 2: 875-877. DOI: 10.1038/nprot.2007.102.

Ali-Shtayeh MS, Yaghmour RM, Faidi YR, Salem K, Al-Nuri MA. 1998. Antimicrobial activity of 20 plants used in folkloric medicine in the Palestinian area. J Ethnopharmacol., 60: 265271. DOI: 10.1016/S0378-8741(97)00153-0.

Ali-Shtayeh MS, Al-Assali AA, Jamous RM. 2013. Antimicrobial activity of Palestinian medicinal plants against acne-inducing bacteria. Afr. J. Microbiol. Res., 7: 2560-2573. DOI: 10.5897/AJMR12.1875.

Al-Mustafa AH, Al-Thunibat OY. 2008. Antioxidant activity of some Jordanian medicinal plants used traditionally for treatment of diabetes. Pak J Biol Sci., 11: 351-358. DOI: 10.3923/pjbs.2008.351.358.

Bar PK, Negoitza D. 2007. The relationships between patch size and Sarcopoterium spinosum association properties at the Mediterranean fringe-Israel. Isr. J. Plant Sci., 55: 93-101. DOI: 10.1560/ijps.55.1.93.

Bayraktar O, Altiok E, Yilmazer Ö, Rusçuklu D, Buyukoz MY. 2016. Antioxidant, antimicrobial and cytotoxic activities of extracts from some selected Mediterranean shrub species (Maquis). Biointerface Res. Appl. Chem., 6: 1437-1444.

Available from: http://biointerfaceresearch.com/?page_id=1475 [10.06.2017].

Bezerra MA, Santelli RE, Oliveira EP, Villar LS, Escaleira, LA. 2008. Response surface methodology (RSM) as a tool for optimization in analytical chemistry. Talanta, 76: 965-997. DOI: 10.1016/j.talanta.2008.05.019.

Choi WY, Jeong MH, Lee HY. 2014. Optimization of extraction process for enhancement of antioxidant activity of Acer mono bark. J Appl Bot Food Qual., 87: 46-55. DOI:10.5073/jabfq.2014.087.007.

Cragg GM, Newman DJ. 2005. Plants as a source of anti-cancer agents. J Ethnopharmacol., 100: 72-79. DOI: 10.1016/j.jep.2005.05.011.

Dibas JI, Yaghi BM, Mansi IA, Mhaidat NM, Al-Abrouni KFS. 2017. Screening for cytotoxic and antioxidant activity of selected wild plants at Shafa Badran, Amman, Jordan. Res. J. Pharm., Biol. Chem. Sci., 8: 489-497. Available from: http://www.rjpbcs.com/pdf/2017_8(2)/[62].pdf [10.06.2017].

Domenico G., Giuseppe F, Piero M., Saverio S, Liliana B. 2007. The status of Sarcopoterium spinosum (Rosaceae) at the western periphery of its range: ecological constraints lead to conservation concerns. Isr J Plant Sci., 55: 1-13. DOI: 10.1560/ijps.55.1.1. 
ElHawary SS, El Shabrawy AER, Ezzat SM, El-Shibani FAA. 2016. Evaluation of the phenolic and flavonoid contents, antimicrobial and cytotoxic activities of some plants growing in $\mathrm{Al}$ Jabal AlAkhdar in Libya. Int J Pharmacognosy and Phytochem Res., 8: 1083-1087. Available from: http://impactfactor.org/ PDF/IJPPR/8/IJPPR,Vol8,Issue7,Article4.pdf [10.06.2017]

Georgios T, Pavlos K, Pantelis X. 2007. Taxonomy and ecology of phryganic communities with Sarcopoterium spinosum (L.)Spach of the Aegean (Greece). Isr J Plant Sci., 55: 15-34. DOI: 10.1560/ijps.55.1.15.

Kasabri V, Afifi FU, Hamdan IV. 2011. In vitro and in vivo acute antihyperglycemic effects of five selected indigenous plants from Jordan used in traditional medicine. J Ethnopharmacol., 133: 888-896. DOI: 10.1016/j.jep.2010.11.025.

Lingzhu L, Lu W, Dongyan C, Jingbo L, Songyi L, Haiqing Y, Yuan Y. 2015. Optimization of ultrasound-assisted extraction of polyphenols from maize filaments by response surface methodology and its identification. J Appl Bot Food Qual., 88: 152-163. DOI: 10.5073/jabfq.2015.088.022.

Lobna KH, Ahmad SA, Talali AA, Mohammad MH. 2013. Chemical Composition of the Essential Oil from Roots of Sarcopoterium spinosum (L.) (Rosaceae) Grown in Syria. J Essent Oil Bear Pl 16: 412-416. DOI: 10.1080/0972060X.2013.813267.

Montgomery DC. 2008. Design and analysis of experiments. Seventh Ed., Place of publication: New York. John Wiley. 978 0-470-12866-4.

Mustafa A, Turner C. 2011. Pressurized liquid extraction as a green approach in food and herbal plants extraction: A review. Anal Chim Acta, 703: 8-18. DOI: 10.1016/j.aca.2011.07.018

Rao MU, Sreenivasulu M, Chengaiah B, Reddy KJ, Chetty CM. 2010. Herbal medicines for diabetes mellitus: a review. Int. J. Pharm Tech Res., 2: 1883-1892. Available from: http://sphinxsai.com/july-sept_2010_vol2.3/pharmtech /pharmtechvol2.3july-sept210/PT=36\%20(1883-1892).pdf.

Re R, Pellegrini N, Proteggente A, Pannala A, Yang M, Rice-Evans C. 1999. Antioxidant activity applying an improved ABTS radical cation decolorization assay. Free Radic Biol Med., 26: 1231-1237. DOI: 10.1016/S0891-5849(98)00315-3.
Reher G, Budesinsky M. 1992. Triterpenoids from plants of the Sanquisorbeae. Phytocemistry, 31: 3909-3914. DOI: 10.1016/S0031-9422(00)97552-6.

Reher G, Reznicek G, Baumann A. 1991-a. Triterpenoids from Sarcopoterium spinosum and Sanguisorba minor. Planta Med., 57: 506. DOI: 10.1055/s-2006-960189.

Reher G, Slijepcevic M, Kraus L. 1991-b. Hypoglycemic activity of triterpenes and tanins from Sarcopoterium spinosum and two Sanguisorba species. Planta Med 57: A57-A58. DOI: 10.1055/s-2006-960318.

Reyes-Munguía A, Carrillo-Inungaray ML, Carranza-Álvarez C, Pimentel-González DJ, Alvarado-Sánchez B. 2016. Antioxidant activity, antimicrobial and effects in the immune system of plants and fruits extracts. Front Life Sci., 9: 90-98. DOI: 10.1080/21553769.2015.1104388.

Ronel M , Malkiel H, Lev-Yadun S. 2007. Quantitative characterization of the thorn system of the common shrubs Sarcopoterium spinosum and Calicotome villosa. Isr J Plant Sci., 55: 63-72. DOI: 10.1560/ijps.55.1.63.

Rosenzweig T, Abitbol G, Taler D. 2007. Evaluating the antidiabetic effects of Sarcopoterium spinosum extracts in vitro. Isr J Plant Sci., 55: 103-109. DOI: 10.1560/ijps.55.1.103.

Singleton VL, Rossi JA. 1965. Colorimetry of total phenolics with phosphomolybdic phosphotungstic acid reagents. Am J Enol Vit., 16: 144-158. Available from: http://www.ajevonline.org /content/16/3/144 [10.06.2017]

Smirin P, Taler D, Abitbol G, Brutman-Barazani T, Kerem Z, Sampson, SR, Rosenzweig T. 2010. Sarcopoterium spinosum extract as an antidiabetic agent: in vitro and in vivo study.J Ethnopharmacol., 129: 10-17. DOI: 10.1016/j.jep.2010.02.021.

Yaniv ZB. 2007. Ethnobotanical studies of Sarcopoterium spinosum in Israel. Isr J Plant Sci., 55: 111-114. DOI: 10.1560/ijps.55.1.111.

Ying X, Wang Y, Jing X, Wenjie Z, Haibo L, Zhang C, Li F. 2009. HPLC determination of eight polyphenols in the leaves of Crataegus pinnatifida Bge. var. major. J. Chromatogr Sci., 47: 201-205. DOI: 10.1093/chromsci/47.3.201. 